\title{
Nucleon electromagnetic form factors from twisted mass lattice QCD
}

\author{
Abdou Abdel-Rehim \\ Computation-based Science and Technology Research Center, The Cyprus Institute, 20 Kavafi \\ Str., Nicosia 2121, Cyprus \\ E-mail: a.abdel-rehimecyi.ac.cy
}

\section{Constantia Alexandrou}

Department of Physics, University of Cyprus, P.O. Box 20537, 1678 Nicosia, Cyprus and Computation-based Science and Technology Research Center, The Cyprus Institute, 20 Kavafi Str., Nicosia 2121, Cyprus

E-mail: alexandeucy.ac.cy

\section{Martha Constantinou}

Department of Physics, University of Cyprus, P.O. Box 20537, 1678 Nicosia, Cyprus

E-mail: marthac@ucy.ac.cy

\section{Kyriakos Hadjiyiannakou}

Department of Physics, University of Cyprus, P.O. Box 20537, 1678 Nicosia, Cyprus

E-mail: hadjigiannakou.kyriakos@ucy.ac.cy

\section{Karl Jansen}

NIC, DESY, Platanenallee 6, D-15738 Zeuthen, Germany

E-mail: karl.jansen@desy.de

\section{Giannis Koutsoulk}

Computation-based Science and Technology Research Center, The Cyprus Institute, 20 Kavafi

Str., Nicosia 2121, Cyprus

E-mail:g.koutsouecyi.ac.cy

\begin{abstract}
Results on the electromagnetic form factors of the nucleon using twisted mass fermion configurations are presented. These include a gauge field ensemble simulated with two degenerate light quarks yielding a pion mass of around $130 \mathrm{MeV}$, as well as two ensembles that include strange and charm quarks in the sea yielding pion masses of $210 \mathrm{MeV}$ and $373 \mathrm{MeV}$. Details of the methods used and systematic errors are discussed, such as noise reduction techniques and the effect of excited states contamination.
\end{abstract}

The 32nd International Symposium on Lattice Field Theory,

23-28 June, 2014

Columbia University New York, NY

\footnotetext{
* Speaker.
} 


\section{Introduction}

The electromagnetic form factors of the proton and neutron are quantities of continuous interest both experimentally and theoretically. Electromagnetic scattering of nucleons reveals properties such as their charge and magnetism, with insight on the distribution of charge within the nucleon. More recently, precision experiments have revealed a surprising discrepancy in the charge radius of the proton. Namely, the proton radius when measured recently via the Lamb shift of muonic hydrogen [1] has a value that is smaller by five standard deviations as compared to experiments using electron scattering and hydrogen Lamb shift [2]. Lattice QCD can provide insight on the origin of this discrepancy by evaluating from first principles the QCD contribution to the proton charge. So far, although consistent across discretisation schemes, lattice simulations at heavier than physical pion masses have underestimated the proton charge [3-5]. With simulations directly at the physical pion mass now becoming available, chiral extrapolations are no longer required, thus eliminating one source of systematic uncertainty and allowing direct contact with experiment. In this proceedings contribution we present preliminary results of the nucleon electromagnetic form factors using three ensembles of twisted mass fermion (TMF) configurations; two $N_{\mathrm{f}}=2+1+1$ ensembles at pion mass $373 \mathrm{MeV}$ and $210 \mathrm{MeV}$ and one $N_{\mathrm{f}}=2$ ensemble at the near physical pion mass value of $130 \mathrm{MeV}$.

\section{Lattice Setup and Methods}

The electromagnetic matrix element of the nucleon can be decomposed into the Dirac $\left(F_{1}\right)$ and Pauli $\left(F_{2}\right)$ form factors:

$$
\left\langle N\left(p^{\prime}, s^{\prime}\right)\left|j^{\mu}\right| N(p, s)\right\rangle=\left(\frac{m_{N}^{2}}{E_{N}\left(\mathbf{p}^{\prime}\right) E_{N}(\mathbf{p})}\right)^{\frac{1}{2}} \bar{u}\left(p^{\prime}, s^{\prime}\right) \mathscr{O}^{\mu} u(p, s), \quad \mathscr{O}^{\mu}=\gamma_{\mu} F_{1}\left(q^{2}\right)+\frac{i \sigma_{\mu v} q^{v}}{2 m_{N}} F_{2}\left(q^{2}\right),
$$

with $m_{N}$ the nucleon mass, $p(s)$ and $p^{\prime}\left(s^{\prime}\right)$ the initial and final momentum (spin) of the nucleon, $u$ are fermion spinors and $q=p^{\prime}-p$ is the momentum transfer. Alternatively one can define the so-called Sachs electric and magnetic form factors: $G_{E}\left(q^{2}\right)=F_{1}\left(q^{2}\right)+F_{2}\left(q^{2}\right)$ and $G_{M}\left(q^{2}\right)=$ $F_{1}\left(q^{2}\right)+\tau F_{2}\left(q^{2}\right)$ with $\tau=\frac{q^{2}}{\left(2 m_{N}\right)^{2}}$. The slope of the form factors at zero momentum defines the relevant radii, namely the Dirac and Pauli radii via: $\left\langle r_{i}^{2}\right\rangle=-\left.\frac{6}{F_{i}} \frac{d F_{i}}{d q^{2}}\right|_{q^{2}=0}$ and similarly for the electric and magnetic radii $\left\langle r_{E}^{2}\right\rangle$ and $\left\langle r_{M}^{2}\right\rangle$.

On the lattice, one calculates an appropriate three-point correlation function:

$$
G^{\mu}\left(\Gamma ; \mathbf{q} ; t_{s}, t_{i}\right)=\sum_{\mathbf{x}_{s} \mathbf{x}_{i}} e^{-i \mathbf{p}^{\prime} \mathbf{x}_{s}} e^{-i\left(\mathbf{p}^{\prime}-\mathbf{p}\right) \mathbf{x}_{i}} \Gamma^{\alpha \beta}\left\langle\bar{\chi}_{N}^{\beta}\left(\mathbf{x}_{s} ; t_{s}\right)\left|j^{\mu}\left(\mathbf{x}_{i} ; t_{i}\right)\right| \chi_{N}^{\alpha}\left(\mathbf{x}_{0} ; t_{0}\right)\right\rangle
$$

with $\chi_{N}$ the nucleon interpolating operators, $x_{s}, x_{i}$ and $x_{0}$ the final (sink), insertion and initial (source) coordinates and the local electromagnetic current: $j^{\mu}(x)=\frac{2}{3} \bar{u}(x) \gamma^{\mu} u(x)-\frac{1}{3} \bar{d}(x) \gamma^{\mu} d(x)$. In this work we use the lattice conserved electromagnetic current [3], and thus lattice results need no renormalisation. In our setup we fix $\mathbf{p}^{\prime}=0$ and carry out the sum over $\mathbf{x}_{s}$ using a sequential inversion through the sink. For this we require $t_{s}-t_{0}$ and the projection matrix $\Gamma$ to be set before the sequential inversion. The choices $\Gamma_{4}=\frac{1}{4}\left(1+\gamma_{4}\right)$ and $\Gamma_{k}=\sum_{j} i \Gamma_{4} \gamma_{5} \gamma_{j}$ isolate $G_{E}$ and $G_{M}$ respectively. 



Figure 1: Diagrams contributing to the nucleon three-point correlation function, connected (left) and disconnected (right).

The three-point correlation function has quark-connected and quark-disconnected contributions as shown in Fig. 1. Here we compute the isovector $(V)$ and isoscalar $(S)$ combinations of the operator: $j_{S}^{\mu}(x)=\bar{u}(x) \gamma^{\mu} u(x) \pm \bar{d}(x) \gamma^{\mu} d(x)$. Assuming SU(2) flavour symmetry the disconnected contribution cancels for the isovector current, but does not in the isoscalar combination. Such disconnected contributions are exceptionally difficult to compute on the lattice due to the increased susceptibility they exhibit to statistical fluctuations. However, recent results using new techniques have shown that at low momentum transfer they are small, of order of $1 \%$, compared to the connected, at pion masses of around 300 to $400 \mathrm{MeV}$ [6, 7]. From the isoscalar and isovector combinations we can obtain the proton and neutron form factors through the linear combinations: $F^{p}-F^{n}=F^{u}-F^{d}$ and $F^{p}+F^{n}=\frac{1}{3}\left(F^{u}+F^{d}\right)$.

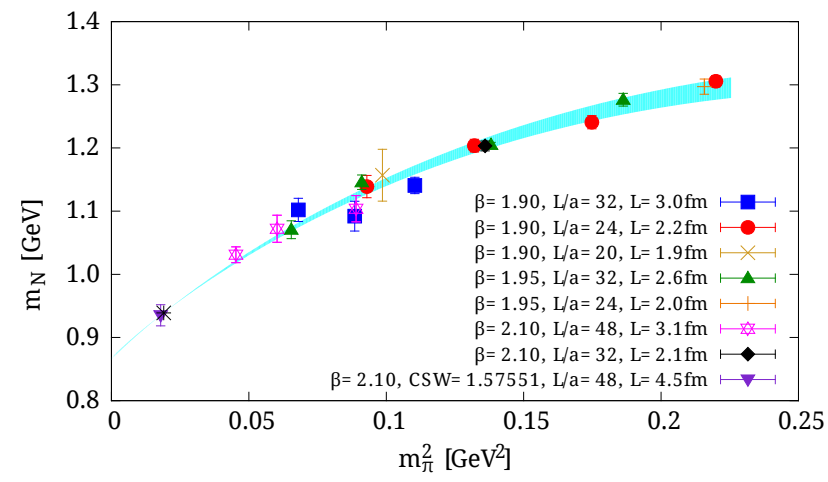

Figure 2: The lattice spacing is set using the nucleon mass.

\begin{tabular}{cccc}
\hline \hline$\beta$ & $L^{3} \times T$ & $\begin{array}{c}a \\
{[\mathrm{fm}]}\end{array}$ & $\begin{array}{c}m_{\pi} \\
{[\mathrm{GeV}]}\end{array}$ \\
\hline \multicolumn{4}{c}{$N_{\mathrm{f}}=2+1+1, c_{\mathrm{SW}}=0$} \\
1.95 & $32^{3} \times 64$ & 0.082 & 0.373 \\
2.10 & $48^{3} \times 96$ & 0.064 & 0.210 \\
\hline \multicolumn{4}{c}{$N_{\mathrm{f}}=2, c_{\mathrm{SW}}=1.57551$} \\
2.10 & $48^{3} \times 96$ & 0.094 & 0.130 \\
\hline \hline
\end{tabular}

Table 1: The twisted mass fermion ensembles used in this work.

In this work we present three ensembles of TMF configurations; two $N_{\mathrm{f}}=2+1+1$ ensembles with $m_{\pi}=373 \mathrm{MeV}$ (referred to as "B55") and $210 \mathrm{MeV}$ (referred to as "D15") and one $N_{\mathrm{f}}=2$ ensemble with $m_{\pi}=130 \mathrm{MeV}$ [8], referred to as the physical point ensemble. More details are given in Table 1. The lattice spacings are determined by fitting the nucleon masses of $18 \mathrm{TMF}$ ensembles using the leading order heavy baryon chiral perturbation theory expansion: $m_{N}=m_{N}^{0}-$ $4 c_{1} m_{\pi}^{2}-\frac{3 g_{A}^{2}}{16 \pi f_{\pi}^{2}} m_{\pi}^{3}$, as shown in Fig. 2. We require the curve to reproduce the physical nucleon mass and allow the spacings to vary as fit parameters. This yields the spacings listed in Table 1 for the ensembles used here.

For extracting the matrix element from the lattice data, we form an appropriate ratio of threepoint to two-point functions, which cancels unknown overlaps and exponential energy factors such that in the limit of large time separations: $t_{i}-t_{0} \gg 1$ and $t_{s}-t_{i} \gg 1$, the contributions from excited nucleon states are damped out, leaving a time-independent ratio (referred to as the plateau region): 

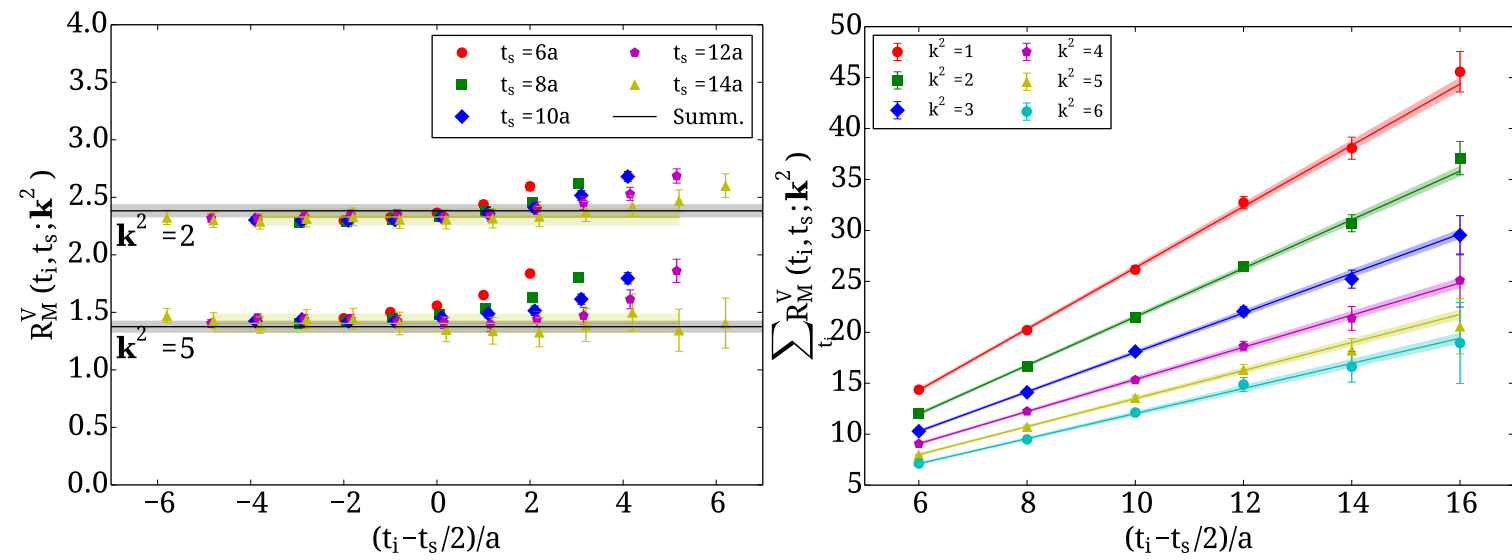

Figure 3: Extraction of the required matrix element is done via fitting to the plateau method (left) or the summation method (right). In the left panel we compare the result of the summation method (black line) with that of the plateau (yellow line) for the B55 ensemble.

$$
R_{M}^{V}\left(t_{i}, t_{s} ; \mathbf{k}^{2}\right) \underset{t_{i}-t_{0} \gg 1}{\frac{t_{s}-t_{i} \gg 1}{t}} G_{M}^{V}\left(\mathbf{k}^{2}\right)\left[1+O\left(e^{-\Delta M\left(t_{s}-t_{i}\right)}, e^{-\Delta E(\mathbf{k})\left(t_{i}-t_{0}\right.}\right)\right]
$$

as illustrated for the isovector magnetic form factor in Fig. 3. $\Delta M(\Delta E)$ is the mass (energy) gap of the nucleon ground to nucleon first exited state. With fixed sink time-slice, we vary the insertion time-slice to identify and fit to the plateau region. This will be referred to as the plateau method. To ensure ground state dominance, we invert for multiple sink-source separations $t_{s}-t_{0}$, with the incremental EigCG algorithm as an efficient multiple right-hand-side solver [9]. With the availability of multiple sink-source separations, we can also sum over the insertion time-slice $t_{i}$ to obtain the summed ratio as a function of $t_{s}$ :

$$
\sum_{t_{i}} R_{M}^{V}\left(t_{i}, t_{s} ; \mathbf{k}^{2}\right) \stackrel{t_{s} \gg 1}{\longrightarrow} C+G_{M}^{V}\left(\mathbf{k}^{2}\right) t_{s}\left[1+O\left(e^{-\Delta M\left(t_{s}-t_{0}\right)}, e^{-\Delta E(\mathbf{k})\left(t_{s}-t_{0}\right)}\right)\right] .
$$

This allows an alternative extraction of the form factor via a two parameter linear fit, the so-called summation method. Compared to the plateau method, the advantage is that excited states decay with a larger exponential suppression factor determined by $t_{s}-t_{0}$ rather than $t_{s}-t_{i}$ or $t_{i}-t_{0}$, while the disadvantage is that a two parameter fit is required. In Fig. 3 we show an example of fits to the summed ratio for momenta up to $\mathbf{k}^{2}=6$.

\section{Results}

In Fig. 4 we show results for the B55 ensemble for which we have carried out inversions at seven sink-source separations for 1,200 configurations. We show the electric and magnetic isovector Sachs form factors obtained via the plateau method and compare them to the summation method to identify excited state effects. As the separation increases from 0.5 to $1.4 \mathrm{fm}$ the form factors become steeper, yielding larger electric and magnetic radii. However these results are still in tension with experiment, shown by the curve which represents J. Kelly's parameterisation of the experimental results. 

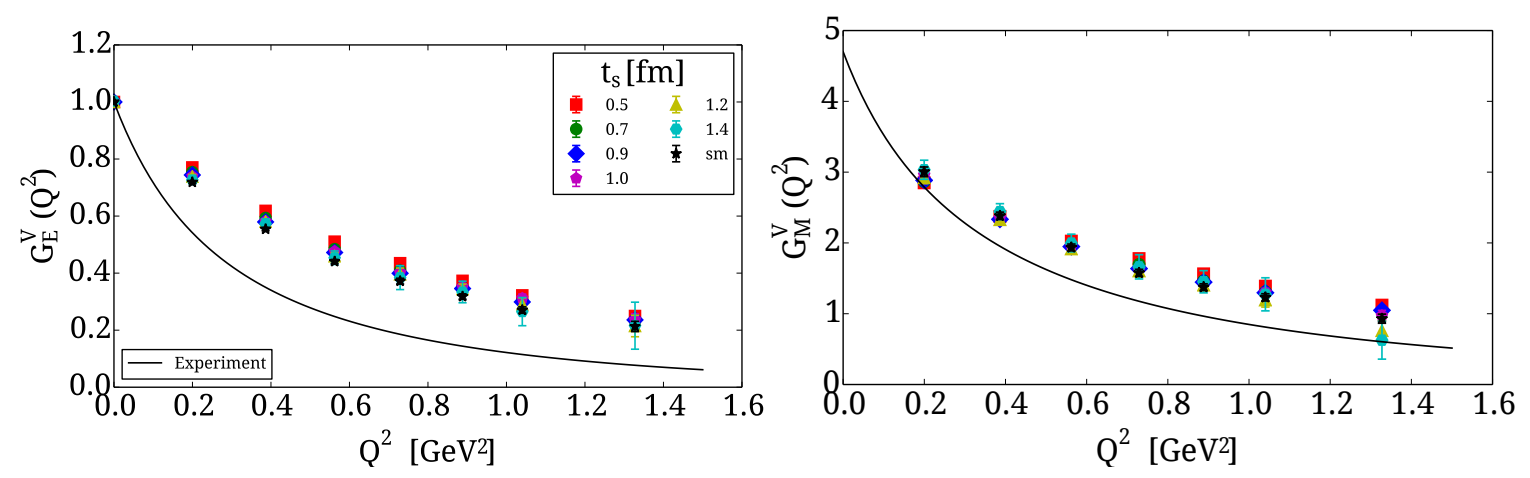

Figure 4: The electric (left) and magnetic (right) nucleon form factors versus the momentum transfer squared, for B55 for various values of $t_{s}$ (coloured symbols) and for the summation method (sm, black asterisk). The solid line is J. Kelly's parameterisation to the experimental data.

The radii are determined from the derivative of the form factors at $Q^{2}=0$. Fitting the form factors to a dipole form $F_{i}\left(Q^{2}\right)=F_{i}(0) /\left[1+Q^{2} / M_{i}^{2}\right]^{2}$ the radii are determined from the dipole mass via $\left\langle r_{i}^{2}\right\rangle=12 / M_{i}^{2}$ with $i=1,2$, and similarly for the Sachs form factors to obtain the electric and magnetic radii. For the Dirac and electric form factors $F_{1}(0)=G_{E}(0)=1$ since we are using the conserved current and thus only the mass is a fit parameter. $F_{2}(0)$ is fitted for and yields the anomalous magnetic moment of the nucleon.
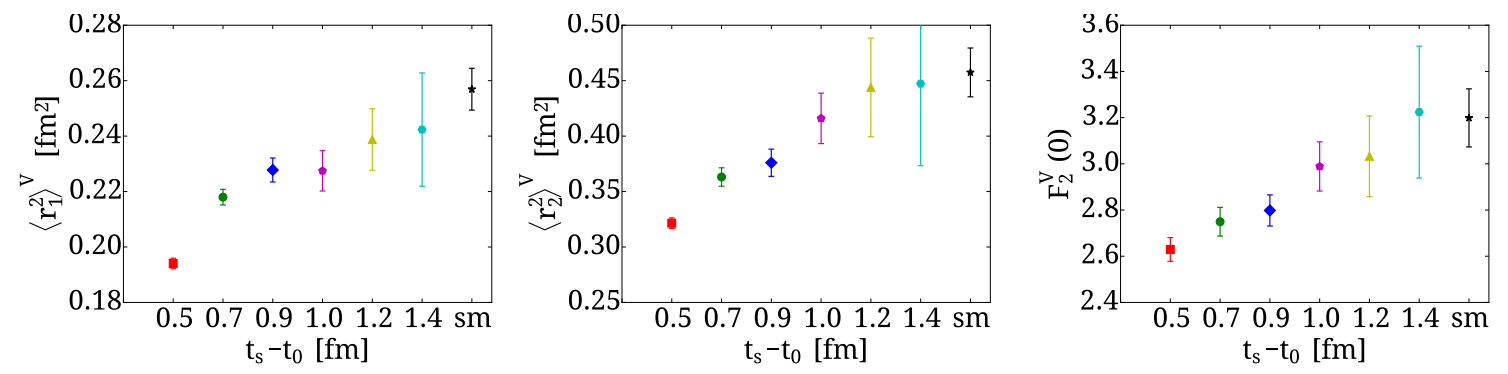

Figure 5: The Dirac and Pauli isovector radii (left and centre), and the isovector magnetic moment (right) for various $t_{s}$ and for the summation method. The notation is the same as in Fig. 4 .

In Fig. 5, we show the isovector Dirac and Pauli radii and the isovector anomalous magnetic moment for the B55 ensemble. Results extracted via fits to the dipole form as a function of the sink-source separations, as well as the value extracted from the summation method. These results corroborate the conclusion that as $t_{s}$ increases the radii become larger. We also observe that contamination from excited states are suppressed after a sink-source separation of around 1.2-1.3 fm, indicated by the agreement between plateau and summation methods beyond this distance.

Our results using all three ensembles, including the physical point ensemble, are shown in Fig. 6, for the isovector electric and magnetic Sachs form factors. A single sink-source separation of $1.2 \mathrm{fm}$ is available for the $m_{\pi}=210 \mathrm{MeV}$ ensemble, while for the physical point we have sinksource separations at $0.94,1.13$ and $1.32 \mathrm{fm}$, the largest of which is shown in Fig. 6, for around 1,400 configurations. We observe a steeper form factor as the pion mass is reduced, although more statistics are required at the physical point for a definite conclusion. In the same figure the electric and magnetic radii are also shown as a function of the pion mass, confirming the tendency towards 

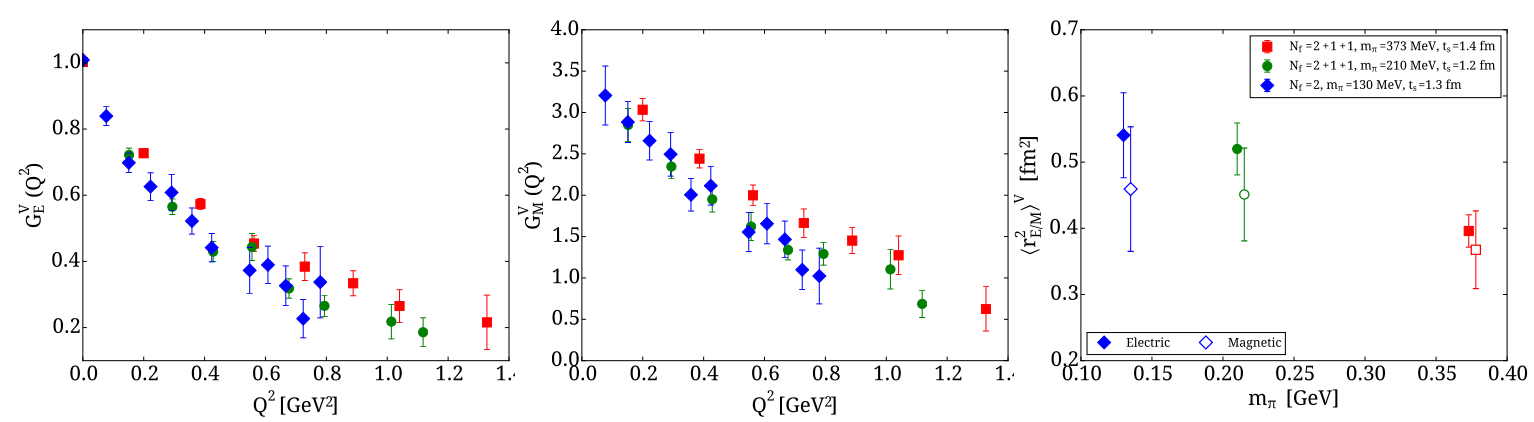

Figure 6: The isovector electric (upper left) and magnetic (lower left) form factors for the three TMF ensembles of this. The associated radii are shown in the right panel as a function of the pion mass.

larger values as the pion mass is decreased.

We compare our results at the physical point with those of a recent calculation using clover fermions at similar pion mass in Fig. 7[ [10]. We see a consistency in the extracted form factors between the two formulations. The isoscalar Pauli form factor $F_{2}^{S}$, not shown in Fig. 7 , is found consistent with zero in both cases.
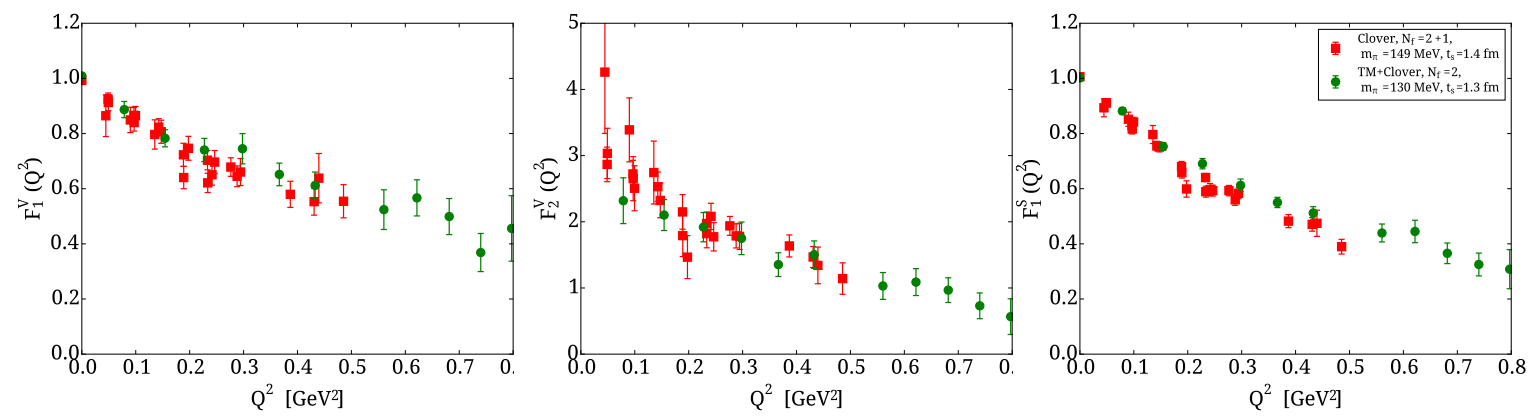

Figure 7: The isovector Dirac (left) and Pauli (centre), and the isoscalar Dirac (right) form factors of this work, compared to results from the LHPC at $m_{\pi}=149 \mathrm{MeV}$ [10].

In Fig. 8 we show the isovector Dirac and Pauli radii as a function of the pion mass, for the TMF ensembles analysed in this work and the clover ensembles analysed in Ref. [10]. We also show the PDG values of these quantities and, for the case of $\left\langle r_{1}^{2}\right\rangle^{V}$, we include the recent result obtained from muonium Lamb shift measurements. As the pion mass decreases the effect of the excited states seem more pronounced indicated by the spread of the open symbols. However, the errors also become larger and a study with increased accuracy is required. Both clover and TMF results are in agreement, and seem to be converging towards the experimental values, although errors are large and need to be drastically reduced to make contact with experiment.

\section{Conclusions}

We have presented preliminary results for the electromagnetic form factors of the nucleon including a lattice QCD ensemble with a pion mass set to its physical value. A lattice at a heavier 

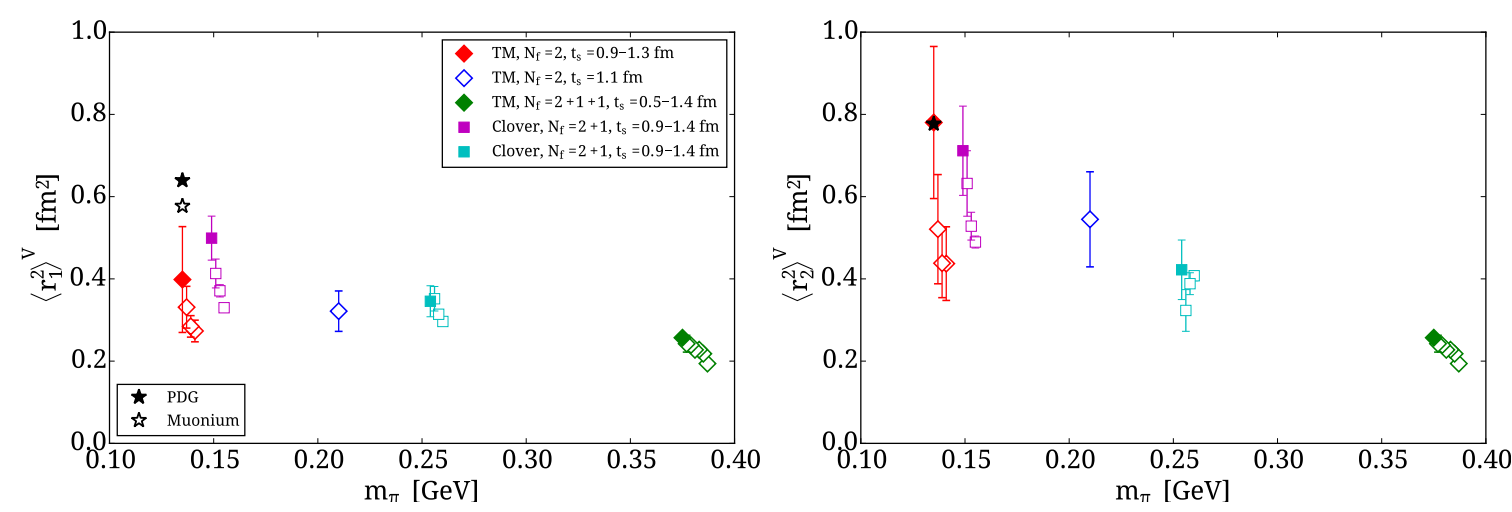

Figure 8: The isovector Dirac (left) and Pauli (right) radii as a function of the pion mass. The diamonds are from this work while the squares are from the LHPC. Filled symbols are results using the summation method, while open symbols are from the plateau method.

pion mass of $373 \mathrm{MeV}$ was used for a thorough study of excited state effects, indicating that a three-point function sink-source separation of $\sim 1.3 \mathrm{fm}$ is sufficient for ensuring ground state dominance. Our results at the physical point are in agreement with recent results using clover fermions at a similar pion mass. Within large statistical errors, our results are either in agreement or tend towards the experimental values as the pion mass is reduced. In order to make contact with experiment, a $2 \%$ error in the radii is required, which amounts to more than a 10 -fold increase in statistics. Deflation algorithms in combination with all-mode-averaging [11] are currently under investigation to efficiently achieve this.

Acknowledgements: M.C., K.H. and K.J. acknowledge support by the Cyprus RPF under con-


EMПЕIPO $2 / 0311 / 16$ respectively. This project used computer time granted by NIC on JUQUEEN (project hch02) and JUROPA (project ecy00) at the JSC as well as by the Cyprus Institute on (project lspro113), under the Cy-Tera project (NEA YПO $\Delta \mathrm{OMH} / \Sigma \mathrm{TPATH} / 0308 / 31$ ).

\section{References}

[1] R. Pohl et al. Nature 466 (2010), pp. 213-216.

[2] P. J. Mohr, B. N. Taylor, and D. B. Newell. Rev.Mod.Phys. 84 (2012), pp. 1527-1605. arXiv: 1203.5425 [physics.atom-ph].

[3] C. Alexandrou et al. Phys.Rev. D83 (2011), p. 094502. arXiv: 1102.2208 [hep-lat].

[4] C. Alexandrou et al. Phys.Rev. D74 (2006), p. 034508. arXiv: hep-lat/ 0605017 [hep-lat ].

[5] S.N. Syritsyn et al. Phys.Rev. D81 (2010), p. 034507. arXiv:0907.4194 [hep-lat].

[6] C. Alexandrou et al. Comput.Phys.Commun. 185 (2014), pp. 1370-1382. arXiv: 1309. 2256 [hep-lat].

[7] S. Meinel et al. PoS Lattice2014 (2014).

[8] A. Abdel-Rehim et al. PoS LATTICE2013 (2013), p. 264. arXiv: 1311.4522 [hep-lat].

[9] A. Abdel-Rehim et al. Phys.Rev. D89.3 (2014), p. 034501. arXiv: 1310.6339 [hep-lat].

[10] J.R. Green et al. Phys.Rev. D90.7 (2014), p. 074507. arXiv: 1404.4029 [hep-lat ]

[11] E. Shintani et al. (2014). arXiv: 1402.0244 [hep-lat]. 